\title{
Detection of enterotoxins and genotyping of Staphylococcus aureus strains isolated from Isfahan Educational Hospital, Iran
}

\author{
Seyed Asghar Havaei, ${ }^{1}$ Nahid Rezaei, ${ }^{2}$ Roholla Havaei, ${ }^{3}$ Amirmorteza Ebrahimzadeh Namvar ${ }^{4}$ \\ ${ }^{1}$ Department of Microbiology, School of Medicine, Isfahan University of Medical Sciences, Isfahan; ${ }^{2}$ Department of \\ Microbiology, Faculty of Bioscience, Falavarjan Branch, Islamic Azad University, Flavarjan; ${ }^{3}$ Department of \\ Endodontics, Zahedan University of Medical Sciences, Zahedan; ${ }^{4}$ Department of Microbiology, Faculty of Medicine, \\ Babol University of Medical Sciences, Babol, Iran
}

\section{Summary}

Background and aims: Staphylococcus aureus is known as one of the most important nosocomial pathogens, which may lead to several infections. The aim of this study was determining the enterotoxins A, C, and TSST-1 and molecular characterization of S. aureus strains with PFGE and MLST typing methods.

Materials and methods: In the present study during the sixmonths sampling, fifty $S$. aureus strains were isolated from patients admitted to Al-Zahra university hospital. Antimicrobial susceptibility testing, Multiplex PCR for detection of enterotoxin A, C and TSST-1, pulse field gel electrophoresis (PFGE) and multilocus sequence typing (MLST) were used for molecular typing.

Results: In antibiogram the highest and lowest percentage of

Correspondence: Amirmorteza Ebrahimzadeh Namvar, Department of Microbiology, Faculty of Medicine, Babol University of Medical Sciences, Babol, Iran.

E-mail: amirmorteza.namvar@gmail.com

Key words: Staphylococcus aureus, Enterotoxin, Molecular Typing, Infection.

Acknowledgments: the authors gratefully acknowledge Amirhossien Ebrahimzadeh Namvar for all supports and contributions to our investigation.

Contributions: the authors contributed equally.

Conflict of interest: the authors declare no potential conflict of interest.

Funding: this study was funded by the Department of Microbiology of Isfahan University of Medical Sciences, Iran.

Received for publication: 29 July 2017.

Revision received: 6 September 2017.

Accepted for publication: 6 September 2017.

CCopyright S.A. Havaei et al., 2017

Licensee PAGEPress, Italy

Microbiologia Medica 2017; 32:6965

doi:10.4081/mm.2017.6965

This article is distributed under the terms of the Creative Commons Attribution Noncommercial License (by-nc 4.0) which permits any noncommercial use, distribution, and reproduction in any medium, provided the original author(s) and source are credited. resistance was belonged to tetracycline and rifampin respectively. Multiplex PCR indicated that $30 \%$ of the strains harbored sea and $34 \%$ harbored sec genes. However, only $4 \%$ of our collected isolates had tsst gene. In PFGE method analysis on all $S$. aureus strains, a total of 19 different patterns were identified. Nine various sequence types in 27 selected $S$. aureus isolates were identified by MLST.

Conclusions: Present study indicates a possible higher variability among our $S$. aureus strains by two different molecular typing methods; nevertheless four main common types (CT1, CT7, CT9, and CT11) with at least one toxin genes were determined.

\section{Introduction}

Staphylococcus aureus is known as one of the most important nosocomial pathogens that can be colonized on the skin of human without any noticeable symptoms and may lead to several infections such as folliculitis, impetigo, cellulitis and food poisoning (9). It is noteworthy that, the staphylococcal infections are created by following the skin barrier failure through surgery and trauma especially in immunocompromised patients. On the other hand the emergence of antibiotic resistant strains, particularly to beta-lactam, aminoglycoside and fluoroquinolone has been considered as a major concern in health care units. The methicillin-resistant $S$. aureus (MRSA) strains are the majority of the mentioned groups due to the rapid spread in health care centres (5). Producing toxins, capsule, various enzymes and enterotoxins are the common virulence factors which have been identified in S. aureus. A large number of $S$. aureus strains are able to produce a family of heat-stable enterotoxins such as staphylococcal enterotoxin serotypes A-E (SEA-SEE) (16).

These toxins are established as superantigens with effect on epithelial cells that subsequently proliferate and stimulate $\mathrm{T}$ cells (12). In comparison to other enterotoxins, enterotoxin A, B and C are responsible for food poisoning $(1,3)$. Moreover, toxic shock syndrome toxin-1 (TSST-1), another secreted toxin by $S$. aureus, which is regarded as a toxic shock syndrome and superantigen, which can bind to MHC-II molecules and stimulating both T cells and Monocytes (8).

According to the spread of $S$. aureus related infections in different parts of hospital and distribution among patients, pulse field gel electrophoresis (PFGE) and multilocus sequence typing (MLST) have been proved as reliable typing methods for $S$. aureus molecular typing.

The aim of this study was determining the enterotoxins A, C, 
and TSST-1 and molecular characterization of $S$. aureus strains with PFGE and MLST typing methods.

\section{Materials and Methods}

\section{Bacterial isolates and culture conditions}

During the six month sampling period, fifty $S$. aureus strains were isolated from patients admitted to Al-Zahra university hospital. The strains were collected from the bloodstream, wound, urine and sputum, thereafter referred to the microbiology laboratory. Each sample was plated onto blood agar with $5 \%$ sheep blood and also nutrient agar and incubated at $35^{\circ} \mathrm{C}$ for $24 \mathrm{~h}$. Standard microbiological tests, including Gram staining, catalase, coagulase and DNase were used to identify the isolates, then stored in trypticase soy broth (TSB) with $15 \%$ glycerol.

\section{Antimicrobial susceptibility testing}

Antimicrobial susceptibility testing was performed on Mueller-Hinton agar, using Kirby-Bauer disc diffusion method with Ciprofloxacin, Gentamicin, Rifampin, Ofloxacin, Tetracycline, Cefoxitin and Cotrimoxazol (Himedia, Mumbai, India) according to CLSI (2017) guidelines.

\section{DNA extraction}

For this purpose, $1.5 \mathrm{~mL}$ of overnight cultured bacteria in TSB was added to $1.5 \mathrm{~mL}$ micro-centrifuge tube and centrifuged at $12000 \mathrm{rpm}$ for $2 \mathrm{~min}$. The supernatant was removed and the pellet resuspended in TE buffer plus lysozyme ( $25 \mathrm{mg} / \mathrm{mL}$ ) (Sigma). The tube was gently shaken and then incubated at $37^{\circ} \mathrm{C}$ for 1 h. $400 \mu \mathrm{L}$ of lysis buffer (Tris-HCL $0.01 \mathrm{M}$, EDTA $0.01 \mathrm{M}$ ), plus $10 \mu \mathrm{L}$ of proteinase $\mathrm{K}$ (Sigma) were added and incubated at $50^{\circ} \mathrm{C}$ for $1 \mathrm{~h}$. Finally the bacterial genome was purified with a commercial kit (K0512 Fermentas) according to the manufacturer's instructions.

\section{Multiplex PCR for detection of enterotoxin A, C and TSST-1}

The presence of genes that encode the enterotoxins A (sea), C (sec) and TSST-1 (tsst) were detected by Multiplex PCR using specific primers, as previously described (13).

\section{PFGE}

All isolates were genotyped by Pulsed-field gel electrophoresis. Genomic DNA was prepared in agarose plugs and digested with $30 \mathrm{U}$ of SmaI (Takara, Japan) as described previously (14). PFGE was carried out in a CHEF-DR III apparatus; (Bio-Rad, USA) at $6 \mathrm{~V} / \mathrm{cm}$ for $22 \mathrm{~h}$ at $12^{\circ} \mathrm{C}$, with pulse times of $5 \mathrm{~s}$ to $50 \mathrm{~s}$. The gels were stained with ethidium bromide and visualized under UV illumination and then photographed. PFGE patterns were analysed by GelCompar II (Applied Maths, Belgium). Salmonella enterica serovar Braenderup strain H9812 was used as a marker.

\section{MLST}

MLST is the powerful molecular typing method which is based on the bacterial housekeeping genes sequence analysis. In the present study MLST was conducted by the method described by Enright et al. (7) The seven housekeeping genes which were used in MLST are as follows: Carbamate kinase ( $\operatorname{arc} C)$, Shikimate dehydrogenase (aroE), Glycerol kinase $(g l p)$, Guanylate kinase $(g m k)$, Phosphate acetyltransferase (pta), Triosephosphate isomerase (tpi) and Acetyl coenzyme A acetyltransferase (yqiL). Allele numbers and sequence types (STs) were assigned according to the S. aureus MLST website (http://saureus. mlst.net). S. aureus strains with positive toxin genes were selected for MLST.

\section{Results}

During the six-month study, various samples were collected from patients admitted to Al-Zahra university hospital. Bloodstream and wound samples had the highest ratio in comparison to other specimens (28\%). Sputum, urine and body fluids were listed in the next stage. Antibiotic susceptibility pattern showed that the highest and lowest percentage of resistance belonged to tetracycline $(42 \%)$ and rifampin (6\%) respectively. Multiplex PCR indicated that $30 \%$ of the strains harbored sea and $34 \%$ harbored $\sec$ genes. However, only $4 \%$ of our collected isolates had tsst gene (Figure 1).

In PFGE method by GelCompar analysis on all S. aureus strains, a total of 19 different patterns were identified. Clinical isolates with the same profiles were grouped in common type (CT) and other clinical isolates with different patterns were grouped in single type. Hence, in PFGE dendrogram 11 CTs (CT1-CT11) and 24 single types were recognized with $90 \%$ similarity cut- off point (Figure 2).

MLST was performed in 27 selected $S$. aureus isolates. Due to the MLST results, nine various sequence types (ST8, ST6, ST22, ST30, ST239, ST1936, ST1937, ST1938, and ST1940) were distinguished (Figure 3 ). The prevalent types were ST8 with $29.6 \%$ and ST22 (14.8\%).

\section{Discussion and Conclusions}

S. aureus is an eminent bacterial pathogen which can cause severe infections. The emergence of resistant strains and the variety of virulence factors such as various toxins and enterotoxins

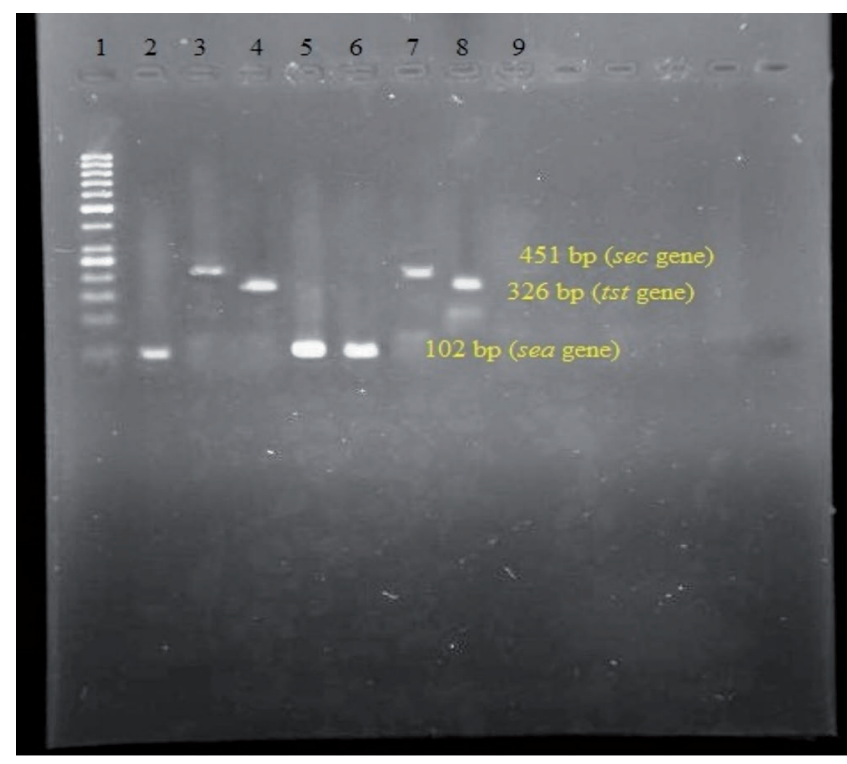

Figure 1. Multiplex PCR amplification products for sea, sec and tst genes of Staphylococcus aureus strains. 
have increased the importance of clinical infections $(2,9)$. Main reasons for these infections are referred to numerous mobile genetic elements such as pathogenicity islands, plasmids and phages (11). Recent study has investigated the prevalence of sea, sec and tsst genes in different clinical samples and also molecular characterization by PFGE and MLST. In our study by Multiplex PCR $30 \%$ and $34 \%$ of strains harboured sea and sec genes respectively. In a study by Hu et al. the $\mathrm{sec}$ gene in addition to others was found to be the most prominent in comparison to other detected toxin genotypes (10). Also in Wang et al. study the sea gene was found with $33 \%$, and followed by sec with $15 \%$. Our results were similar to other investigators, however the percentage of sea and sec genes in some studies are different (20).
The prevalence of the tsst gene in our studied isolates was found to be $4 \%$, although this ratio in other studies was different. As such, in a study by El-Ghodban et al. in Libya $7.5 \%$ of clinical strains and none of the food strains harboured the tsst gene (6). Likewise the percentage of this gene in the study conducted by Tsen et al. (1998) in Taiwan was reported to be $4.8 \%$. The positive strains were also sec producers (17). The apparent issue in this regard is that the most clinical $S$. aureus strains contained at least one staphylococcal enterotoxin genes (19). The various reported percentages of toxin genes in different studies may be dependent on the frequency of these genes in different sources and special strains such as epidemic and multidrug resistant strains or the horizontal transferring of mobile genetic elements between these

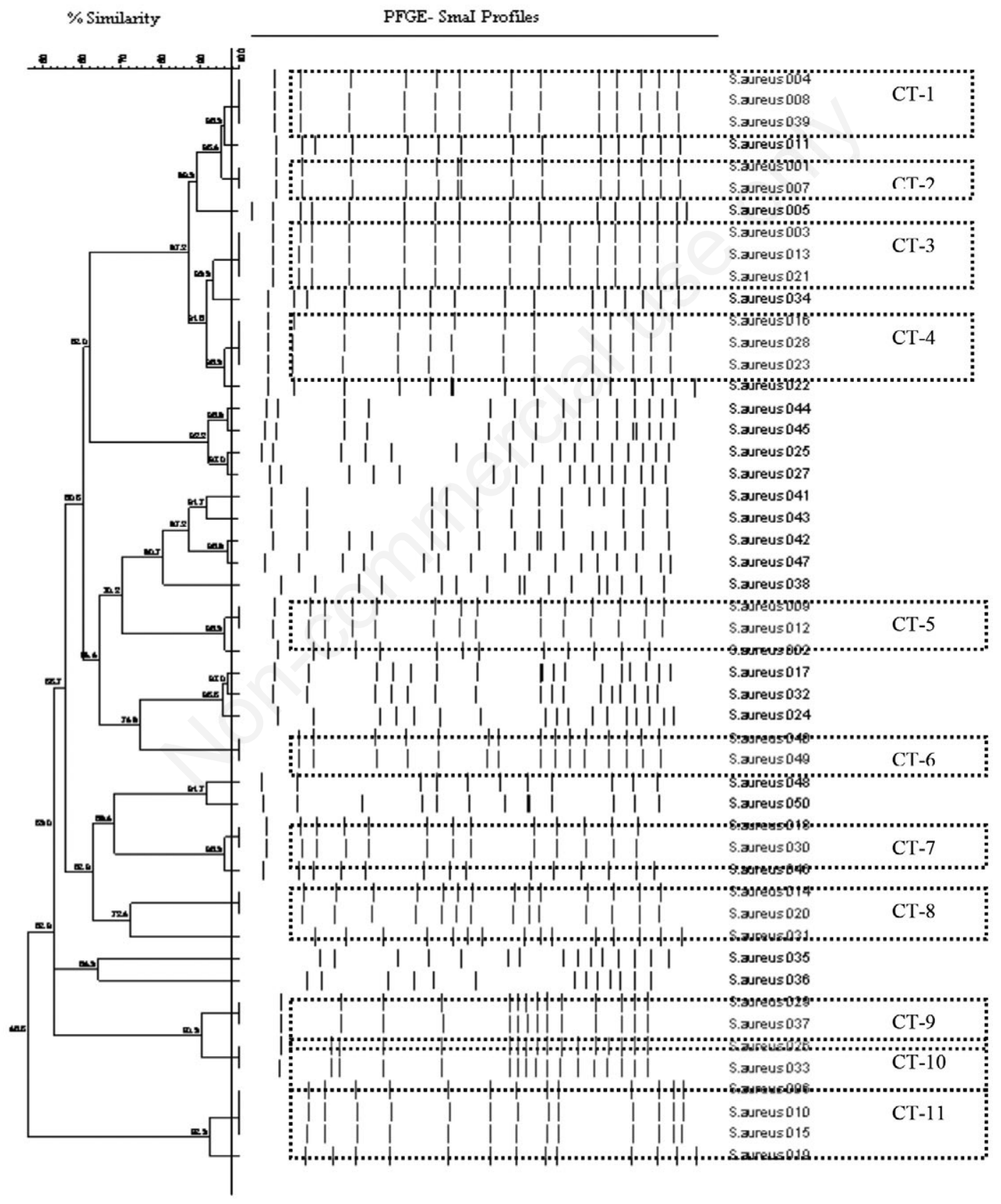

Figure 2. Pulsed-field gel electrophoresis dendrogram of $50 \mathrm{~S}$. aureus strains, with a $90 \%$ similarity cut-off point clustered by unweighted pair grouping by mathematical averaging. 


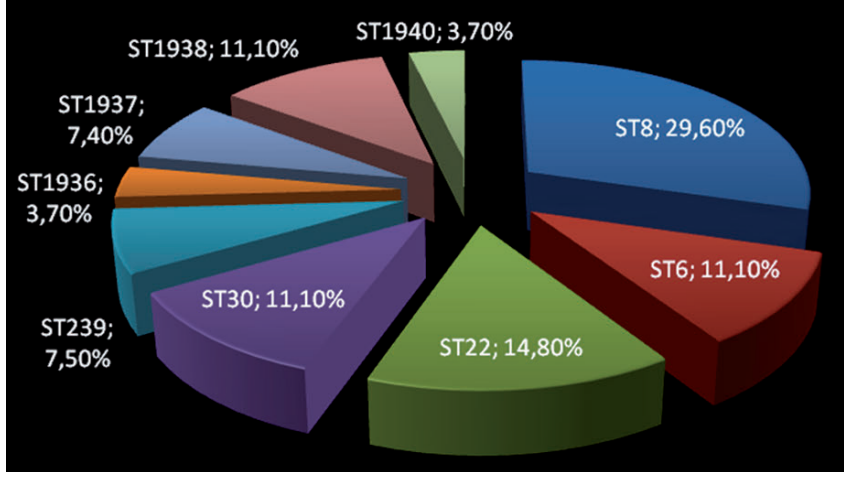

Figure 3. The frequency of sequence types (STs) in S. aureus strains isolated from Al-Zahra University Hospital, Iran.

strains. Peck and colleagues reported that the $71.4 \%$ of $S$. aureus strains harboured the enterotoxin genes (15).

In antimicrobial susceptibility test, the majority of our studied strains showed susceptibility to all antibiotics except tetracycline (42\%). Among molecular typing techniques, PFGE is used as a primary and the gold standard method for $S$. aureus surveillance and epidemiological studies. However, MLST is used for more detailed analysis and outbreak investigations $(4,18)$. Using pulsed field gel electrophoresis, nineteen different patterns were identified with twenty four single types. PFGE results demonstrated the genetic heterogeneity in our collected $S$. aureus isolates, albeit the PFGE method confirmed the complementary information in the present study. Thus strains which harboured both sea and sec genes were classified in CT4, CT7 and CT9, while tsst gene positive strains were sorted in CT1 and CT11 respectively. On the other hand the strains with sea and tsst genes were categorized in CT1.

In MLST typing from the nine recognized STs, ST8 was the widespread type (detected in eight of 27 strains), and thereafter ST22. In MLST, the ST8 and ST22 strains were classified as CT1, CT6, CT7, CT9, and CT11 in PFGE dendrogram.

In conclusion the present study indicates a possible higher variability among our $S$. aureus strains by two different molecular typing methods; nevertheless four main common types (CT1, CT7, CT9, and CT11) with at least one toxin genes were determined.

\section{References}

1. Akineden O, Hassan AA, Schneider E, Usleber E. Enterotoxigenic properties of Staphylococcus aureus isolated from goats' milk cheese. Int J Food Microbiol 2008;124:2116.

2. Becker K, Friedrich AW, Lubritz G, et al. Prevalence of genes encoding pyrogenic toxin superantigens and exfoliative toxins among strains of Staphylococcus aureus isolated from blood and nasal specimens. J Clin Microbiol 2003;41:1434-9.

3. Chapaval L, Moon DH, Gomes JE, et al. Use of per to detect classical enterotoxins genes (ent) and toxic shock syndrome toxin-1 gene (tst) in Staphylococcus aureus isolated from crude milk and determination of toxin productivities of S. aureus isolates harboring these genes. Arq Inst Biol 2006;73:165-9.

4. Cookson BD, Robinson DA, Monk AB, et al. Evaluation of molecular typing methods in characterizing a European collection of epidemic methicillin-resistant Staphylococcus aureus strains: the HARMONY collection. J Clin Microbiol 2007;45:1830-7.

5. Ebrahimzadeh Namvar A, Afshar M, Asghari B, Rastegar Lari A. Characterisation of SCCmec elements in methicillin-resistant Staphylococcus aureus isolated from burn patients. Burns 2013;40:708-12.

6. El-Ghodban A, Ghenghesh KS, Marialigeti K, Esahli H, Tawil A. PCR detection of toxic shock syndrome toxin of Staphylococcus aureus from Tripoli, Libya. J Med Microbiol 2006;55:179-82.

7. Enright MC, Day NP, Davies CE, et al. Multilocus sequence typing for characterization of methicillin-resistant and methicillin susceptible clones of Staphylococcus aureus. J Clin Microbiol 2000;38:1008-15.

8. Fernandez MM, Marzi MC, Berguer P, et al. Binding of natural variants of staphylococcal superantigns SEG and SEI to TCR and MHC class II molecule. Mol Immunol 2006;43:927-38.

9. Holtfreter S, Grumann D, Schmudde M, et al. Clonal distribution of superantigen genes in clinical Staphylococcus aureus isolates. J Clin Microbiol. 2007;45:2669-80.

10. Hu D, Omoe K, Inoue F, et al. Comparative prevalence of superantigenic toxin genes in meticillin-resistant and meticillin susceptible Staphylococcus aureus isolates. J Med Microbiol 2008;57:1106-12.

11. Jarraud S, Mougel C, Thioulouse J, et al. Relationships between Staphylococcus aureus genetic background, virulence factors, age groups (alleles), and human disease. Infect Immunol 2002;70:631-41.

12. Llewelyn M, Cohen J. Superantigens: microbial agents that corrupt immunity. Lancet Infect Dis 2002;2:156-62.

13. Mehrotra M, Wang G, Johnson WM. Multiplex PCR for detection of genes for Staphylococcus aureus enterotoxins, exfoliative toxins, toxic shock syndrome toxin 1, and methicillin resistance. J Clin Microbiol 2000;38:1032-5.

14. Murchan S, Kaufmann ME, Deplano A, et al. Harmonization of pulsed-field gel electrophoresis protocols for epidemiological typing of strains of methicillin-resistant Staphylococcus aureus: a single approach developed by consensus in 10 European laboratories and its application for tracing the spread of related strains. J Clin Microbiol 2003;4:1574-85.

15. Peck KR, Baek JY, Song JH, Ko KS. Comparison of genotypes and enterotoxin genes between Staphylococcus aureus isolates from blood and nasal colonizers in a Korean Hospital. J Korean Med Sci 2008;24:585-91.

16. Pereira V, Lopes C, Castro A, et al. Characterization for enterotoxin production, virulence factors, and antibiotic susceptibility of Staphylococcus aureus isolates from various foods in Portugal. Food Microbiol 2009;26:278-82.

17. Tsen HY, Yu GK, Wang KC, et al. Comparison of enterotoxigenic types, toxic shock syndrome toxin 1 (TSST-1) strains and antibiotic susceptibilities for enterotoxigenic Staphylococcus aureus strains isolated from food and clinical samples. Food Microbiol 1998;15:33-41.

18. Vainio A, Kardén-Lilja M, Ibrahem S, et al. Clonality of epidemic methicillin-resistant Staphylococcus aureus strains in Finland as defined by several molecular methods. Eur J Clin Microbiol Infect Dis 2008;27:545-55.

19. Varshne A, Mediavilla J, Robiou N, et al. Diverse Enterotoxin Gene Profiles among Clonal Complexes of Staphylococcus aureus Isolates from the Bronx, New York. Appl Environ Microbiol 2009;75:6839-49.

20. Wang LX, Hu ZD, Hu YM, et al. Molecular analysis and frequency of Staphylococcus aureus virulence genes isolated from bloodstream infections in a teaching hospital in Tianjin, China. Genet Mol Res 2013;12:646-54. 